\title{
Using Syndromic Surveillance to Quantify ED Visits for Coagulopathy Cases
}

\section{Kelly Walblay, Megan Patel, Stacey Hoferka}

Communicable Diseases, Illinois Department of Public Health, Chicago, Illinois, United States

\section{Objective}

To determine whether emergency department (ED) visits were captured in syndromic surveillance for coagulopathy cases associated with an outbreak linked to synthetic cannabinoid (SC) use and to quantify the number of ED visits and reasons for repeat visits.

\section{Introduction}

In March 2018, the Illinois Department of Public Health (IDPH) was informed of a cluster of coagulopathy cases linked to SC use. By June 30, 2018, 172 cases were reported, including five deaths, where 74\% were male and the mean age was 35.3 years (range: 18-65 years). All cases presented to an emergency department (ED) at least once for this illness. Ninety-four cases provided clinical specimens and all tested positive for brodifacoum, a long-acting anticoagulant used in rodenticide. Cases were reported to the health department by the Illinois Poison Control Center and direct reporting from hospitals. REDCap was the primary database for tracking cases and collecting demographic information, risk factor data and healthcare facility utilization, including number of ED visits.

Syndromic surveillance was utilized to monitor ED visits related to the cluster, assist with case finding and provide situational awareness of the burden on the EDs and geographic spread. In this study, we retrospectively used syndromic surveillance along with the data in REDCap to quantify the number of ED visits per coagulopathy case, understand the reasons for repeat visits, and determine whether visits were captured in syndromic surveillance.

\section{Methods}

Illinois hospital ED data submitted to the National Syndromic Surveillance Platform instance of ESSENCE (ESSENCE), was compared to data present in our primary REDCap database. A subset of the cases, males $18-44$ years of age $(n=105 ; 61 \%$ of cases), were included in this analysis. Illinois ESSENCE data in males aged 18-44 years from March 10, 2018-June 30, 2018 were matched to cases in the REDCap database by age, zip code, initial visit date, facility, and reason for visit including: chief complaint, discharge diagnosis, and triage note. If the initial visit was found, the matching criteria and medical record number were used to search for additional related visits. The number of visits in ESSENCE and reasons for visits were totaled for each patient. Reasons for repeat visits were categorized into four categories: continued gross bleeding or symptoms associated with coagulopathy, medical evaluation or follow-up, laboratory work and prescription refill. Repeat visits may fall into more than one category. The number and dates of ED visits captured in ESSENCE per case were compared to that reported in REDCap. An epidemic curve was constructed to display the number of ED visits and type (i.e. primary visit or repeat visit) captured by REDCap only, ESSENCE only or both by visit date.

\section{Results}

Of the 105 cases in REDCap, 89 (85\%) were matched to at least one ED visit in ESSENCE from March 10, 2018-June 30, 2018. The mean number of ESSENCE ED visits per case was 1.9 visits and the median was one visit (range: $1-11$ visits). The main chief complaints for the primary visit included hematuria $(n=31)$, abdominal pain $(n=20)$, back pain/flank pain $(n=13)$, K2 $(n=11)$, bleeding from multiple sites $(n=8)$, vomiting blood $(n=7)$, and urinary tract infection or kidney stones $(n=7)$. Of the 89 cases matched to a visit in ESSENCE, 84 (94\%) cases, representing 142 (79\%) of ED visits, were captured by syndrome definitions that were being utilized to monitor the cluster. Forty-three cases (48\%) had at least two visits in ESSENCE. The reasons for return visits captured in ESSENCE $(n=84)$ were continued gross bleeding or symptoms as sociated with coagulopathy $(n=53)$, medical evaluation or follow-up $(n=14)$, laboratory work $(n=13)$, prescription refill $(n=7)$ or unknown $(n=2)$. Of the 105 cases, the number of ED visits reported in REDCap matched the number of visits found in ESSENCE for 49 cases $(47 \%)$. For 24 cases (23\%), ESSENCE identified more visits than REDCap and for 16 cases (15\%), REDCap had more ED visits reported than captured in ESSENCE. Sixteen cases (15\%) in REDCap were not found in ESSENCE. All of the unmatched visits were due to ESSENCE data quality, including a lack of reporting hospital admissions, lack of submitting data to ESSENCE, and missing data including: date of birth, medical record number, and triage notes.

SDS Annual Conference Proceedings 2019. This is an Open Access article distributed under the terms of the Creative Commons AttributionNoncommercial 3.0 Unported License (http://creativecommons.org/licenses/by-nc/3.0/), permitting all non-commercial use, distribution, and reproduction in any medium, provided the original work is properly cited. 


\section{Conclusions}

Syndromic surveillance was a useful tool in describing the burden of ED visits for patients in the Illinois coagulopathy outbreak linked to SC use. ESSENCE data helped to quantify the number of ED visits per patient and identify patients that re-presented for the same illness. The most common reason for repeat ED visits was continued symptoms, which may be attributed to misdiagnosis at the initial healthcare visit. ED visits that were not picked up by ESSENCE were a result of data quality issues from select facilities that were not reporting hospitalizations or key information such as date of birth, medical record number or triage notes. Engagement with healthcare facilities to provide this information will improve the data quality of syndromic surveillance.

\section{Acknowledgement}

This study was supported in part by an appointment to the Applied Epidemiology Fellowship Program administered by the Council of State and Territorial Epidemiologists (CSTE) and funded by the Centers for Disease Control and Prevention (CDC) Cooperative Agreement Number 1U38OT000143-05.

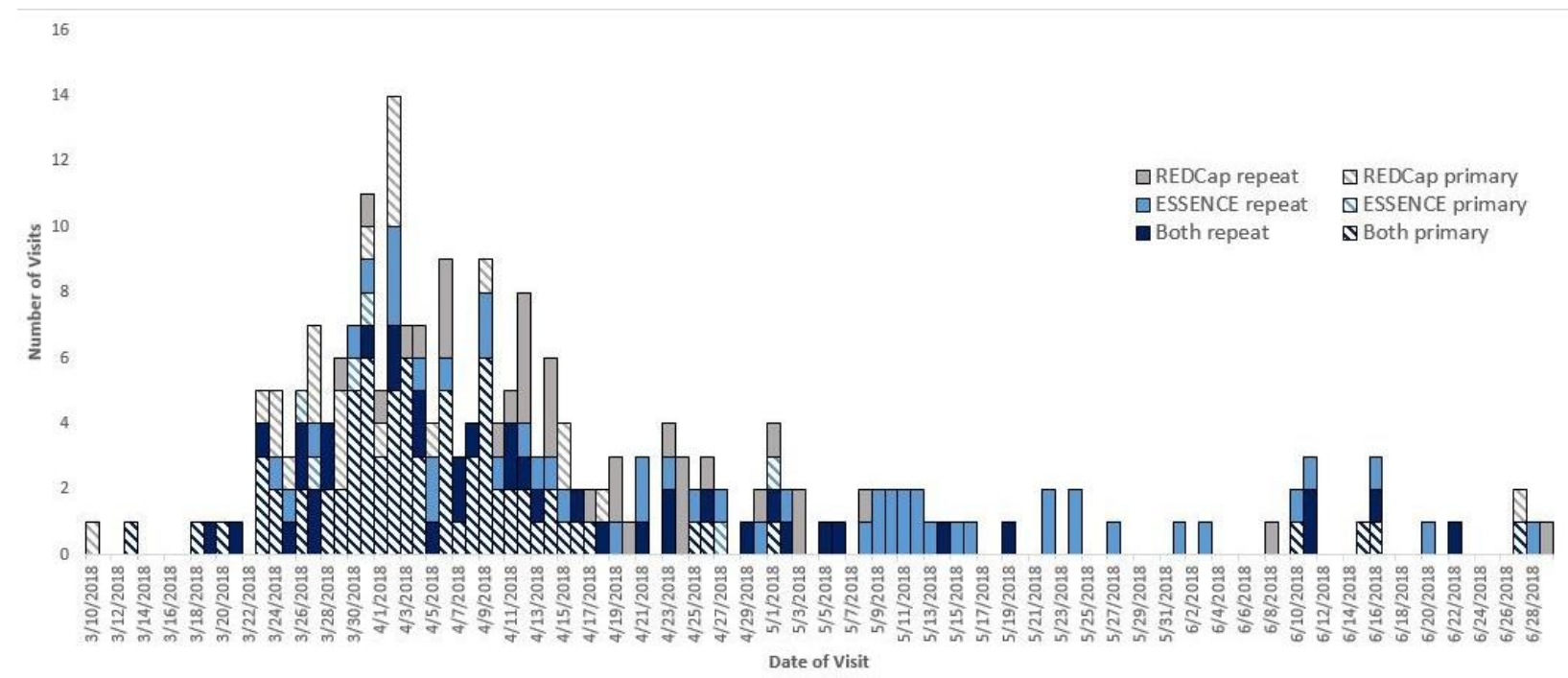

Figure 1. Emergency Department (ED) Visits for Coagulopathy Cases by Surveillance System and Type of Visit, Illinois, March 10-June 30, 2018 